Published in final edited form as:

Gait Posture. 2013 May ; 38(1): . doi:10.1016/j.gaitpost.2012.10.006.

\title{
Hearing Loss and Gait Speed Among Older Adults in the United States
}

\author{
Lingsheng Li, M.H.S. ${ }^{1,2}$, Eleanor M. Simonsick, Ph.D. ${ }^{3}$, Luigi Ferrucci, M.D. Ph.D. ${ }^{3}$, and \\ Frank R. Lin, M.D. Ph.D. $1,2,4$ \\ ${ }^{1}$ Center on Aging and Health, Johns Hopkins Medical Institutions, Baltimore, Maryland \\ ${ }^{2}$ Department of Otolaryngology-Head \& Neck Surgery, Johns Hopkins School of Medicine, \\ Baltimore, Maryland ${ }^{3}$ Longitudinal Studies Section, Clinical Research Branch, National Institute \\ on Aging, Baltimore, Maryland ${ }^{4}$ Department of Epidemiology, Johns Hopkins Bloomberg School \\ of Public Health, Baltimore, Maryland
}

\begin{abstract}
Background-Previous studies have suggested that hearing loss, which is highly prevalent but undertreated in older adults, may be associated with gait and physical functioning. Determining if hearing loss is independently associated with gait speed is critical toward understanding whether hearing rehabilitative interventions could help mitigate declines in physical functioning in older adults.
\end{abstract}

\begin{abstract}
Methods-We analyzed cross-sectional data from the 1999-2002 cycles of the National Health and Nutritional Examination Survey during which participants 50-69 years $(\mathrm{n}=1180)$ underwent hearing and gait speed assessments. Hearing was defined by a pure tone average of hearing thresholds at $0.5-4 \mathrm{kHz}$ tones in the better-hearing ear. Gait speed was obtained in a timed 20-foot (6.1 meter) walk. Linear and logistic regression models were used to examine the association between hearing loss and gait speed while adjusting for demographic and cardiovascular risk factors. Analyses incorporated sampling weights to yield results generalizable to the U.S. population.
\end{abstract}

Results-In a model adjusted for demographic and cardiovascular risk factors, a $25 \mathrm{~dB}$ hearing loss was associated with slower gait speed $(-0.05 \mathrm{~m} / \mathrm{s}$ per $25 \mathrm{~dB}$ HL [95\% CI: $-0.09--0.02])$ and a nearly two-fold increased odds of having a gait speed $<1.0 \mathrm{~m} / \mathrm{s}$ (OR 2.0, 95\% CI: $1.2-3.3$ ). The reduction in gait speed associated with a $25 \mathrm{~dB}$ hearing loss was equivalent to that associated with an age difference of nearly 12 years.

Conclusions-Greater hearing loss is independently associated with slower gait speed. Further studies investigating the mechanistic basis of this association and whether hearing rehabilitative interventions could affect gait and physical functioning are needed.

\footnotetext{
(C) 2012 Elsevier B.V. All rights reserved.

Address correspondence, reprint requests, and proofs to: Frank R. Lin, Johns Hopkins Center on Aging \& Health, 2024 E. Monument St., Suite 2-700, Baltimore, MD 21205, Telephone: (443) 287-6509, Fax: (410) 502-6713, flin1 @ jhmi.edu.

Publisher's Disclaimer: This is a PDF file of an unedited manuscript that has been accepted for publication. As a service to our customers we are providing this early version of the manuscript. The manuscript will undergo copyediting, typesetting, and review of the resulting proof before it is published in its final citable form. Please note that during the production process errors may be discovered which could affect the content, and all legal disclaimers that apply to the journal pertain.

All authors report no financial or personal conflicts of interest.
} 


\section{Introduction}

Walking constitutes a complex task that requires the coordinated functioning of multiple organ systems, and, therefore, gait speed is considered a robust indicator of health status $[1,2]$. Previous studies have explored the roles of biological, cognitive, and social determinants in predicting gait speed decline. Increasingly, measures of cognitive performance including executive functioning have been found to be associated with gait speed [3-5].

While hearing loss has not been previously considered a risk factor for slower gait speed, hearing loss is associated with several conditions such as increased cognitive load [6], poorer executive functioning [7,8], and reduced social and physical functioning [9] that could plausibly promote slower gait speed. Alternatively, hearing loss and gait speed may be linked through concomitant cochlear and vestibular dysfunction or a shared pathologic etiology such as from microvascular disease. Hearing loss is highly prevalent [10] yet remains vastly undertreated in older adults [11]. Determining if hearing loss is independently associated with gait speed is an important first step toward understanding whether hearing rehabilitative interventions could possibly help mitigate declines in physical functioning.

The objective of the current study was to examine the cross-sectional association between hearing loss and gait speed in a nationally-representative sample of adults aged 50-69 years. We investigated whether greater hearing loss was associated with reduced gait speed obtained in a 20 -foot (6.1 meter) timed walk. We hypothesized a priori that greater hearing loss as measured by pure tone audiometry is associated with slower gait speed after adjustment for potential confounders.

\section{Methods}

\section{Study subjects}

Subjects for this study were participants (age 50-69 years) who completed both the audiometric examination and timed 20-foot walk in the 1999-2002 cycles of the National Health and Nutritional Examination Survey (NHANES), an ongoing program of studies designed to assess the health, functional, and nutritional status of the United States civilian non-institutionalized population. From 1999-2002, audiometry was administered to a half sample of all adults 20-69 years, and gait speed was assessed in participants 50 years and older. No subsequent NHANES cycles have concurrently assessed both hearing loss and gait speed. Each sequential cross-sectional study in NHANES uses a stratified, multistage probability sampling design to survey a sample of the population, with selective oversampling of low-income individuals, racial minorities, and older adults [12]. Sampling weights allow for analyses that account for the complex sampling survey and yield results that are generalizable to the U.S. population. De-identified data available within public domain, as provided by NHANES, were used for the study. The NHANES protocol (\#98-12) was reviewed and approved by the National Center for Health Statistic's Institutional Review Board (IRB) and documented informed consent was obtained from all participants.

\section{Audiometric assessment}

Audiometry was performed by a trained examiner according to established NHANES protocols [13]. Briefly, a trained examiner obtained air conduction hearing thresholds from both ears in a dedicated, sound-isolating room in a mobile examination center. Testing was conducted according to a modified Hughson Westlake procedure using the automated testing mode of the audiometer (Interacoustics AD226) and/or manually per the testing protocol. 
Quality assurance and quality control were established through daily calibration of equipment and monitoring of ambient noise levels using a sound level meter. The audiometric test room met or exceeded ANSI S3.1-1991 guidelines for maximum permissible ambient noise levels. Air conduction stimuli were presented primarily through supra-aural earphones (TDH 39P). Insert earphones (ER3A) were reserved for cases of collapsing ear canals or for a cross-over retesting protocol in cases of asymmetric hearing loss (masking was not performed). As an additional quality measure, thresholds were measured twice at $1 \mathrm{kHz}$ in both ears, and audiometry was repeated if there was $>10 \mathrm{~dB}$ discrepancy between the threshold measurements. We utilized hearing thresholds from 0.5$4 \mathrm{kHz}$, using the first threshold tested at $1 \mathrm{kHz}$ and incorporating manual re-test thresholds as needed. Hearing loss was defined as a speech-frequency pure tone average (PTA) of thresholds at $0.5,1,2$, and $4 \mathrm{kHz}$ in the better hearing ear in accordance with the World Health Organization [14]. Categories of hearing loss severity were based on American Speech-Language Hearing Association guidelines [15], but several categories were collapsed to simplify analyses (Normal hearing $\leq 25 \mathrm{~dB}$, Mild loss $>25 \mathrm{~dB}$ and $\leq 40 \mathrm{~dB}$, Moderate loss $>40 \mathrm{~dB}$ and $570 \mathrm{~dB}$, Severe loss $>70 \mathrm{~dB}$ ). All hearing thresholds are reported as $\mathrm{dB}$ HL, which is a standard audiometric measurement used to assess the level of hearing loss.

\section{Gait Speed Assessment}

Gait speed was obtained with a timed 20 -foot (6.1 meter) walk, as administered per the NHANES protocol [16]. Briefly, participants were instructed to walk at their usual pace, with the use of a walker or cane if needed, along a designated 20-foot long test track area in the MEC. A certified health technician used a hand-held stopwatch to measure the time from when the participant's first foot touched the floor beyond the start line to when his or her foot touched the floor across the 20-foot finish line. Gait speed was calculated by dividing the walking distance ( 20 feet $=6.10$ meters) by the time (seconds) needed to complete the walk. Individuals with a history of myocardial infarction within the past six weeks, chest or abdominal surgery within the past three weeks, knee surgery or knee replacement surgery, severe back pain, a history of brain aneurysm or stroke, and/or inability to walk without holding onto someone were excluded from gait speed testing. Quality assurance and quality control were established through monitored site visits, procedural checklists, and continuous data review for identifying systematic errors.

\section{Covariates}

Data on demographic variables and medical history were obtained from interviews. Selfreported race/ethnicity was grouped as Mexican-American/other Hispanic (Hispanic), nonHispanic white (white), non-Hispanic black (black), or other race. Education was collapsed into a 4 level variable (less than high school, high school graduate, some college, or college graduate).We adjusted for race and education because they may be associated with both hearing loss and health status and therefore could be potential confounders. Hearing aid use was based on whether an individual with hearing loss reported using a hearing aid at least once a day over the preceding year. Variables related to medical history included diabetes (based on self-reported diagnosis and/or current use of insulin or other diabetic medications), smoking (current/former/never), hypertension (told by physician on two or more visits about hypertension diagnosis), and stroke (self-reported history).

\section{Statistical Methodology}

We accounted for the complex sampling design in all analyses by using sample weights according to National Center for Health Statistics (NCHS) guidelines [17] except for Table 1. The purpose of Table 1 was only to give descriptive statistics on the characteristics of the study cohort rather than nationally generalizable estimates and hence weights were not used. 
Locally weighted scatterplot smoothing (lowess) was used to graphically explore the association of hearing loss and age with gait speed and to identify non-linear data trends and outliers. Based on the lowess plot, two participants with gait speed $>2 \mathrm{~m} / \mathrm{s}$ were identified as outliers and were excluded from further analyses. We also excluded participants with gait speed $<0.6 \mathrm{~m} / \mathrm{s}(\mathrm{n}=40)$ to limit the analysis to non-frail individuals [18]. We used linear and logistic regression models to analyze whether hearing loss was associated with slower gait speed, after adjusting for age and other covariates. The $\beta$-coefficients from linear regressions are interpreted as the average difference in gait speed (i.e. negative values indicate slower gait speed) associated per unit change in hearing loss and age. A sensitivity analyses was also performed to normalize the gait speed variable with respect to standing height (normalized speed $=$ speed $\times 1 / \sqrt{ }$ (Height $\times \mathrm{g}$ ), where height is measured in meters and $\mathrm{g}=$ $\left.9.81 \mathrm{~m} / \mathrm{s}^{2}\right)[19,20]$. Per NCHS guidelines, the Taylor Series Linearization method was used for variance estimation. All analyses were conducted using STATA 12 (StataCorp, College Station, TX).

\section{Results}

Table 1 summarizes the demographic characteristics of the study population. From 19992002, 1180 participants aged 50-69 years underwent concurrent assessments of hearing loss and gait speed in NHANES. Hearing loss $>25 \mathrm{~dB}$ was prevalent in $23.0 \%$ of these participants, and among those with hearing loss, hearing aids were used by $4.4 \%$. The vast majority of participants had hearing thresholds in the normal-to-mild range with only $5.2 \%$ of participants having a moderate or greater hearing loss. The mean gait speed was 1.05 $(\mathrm{SD}=0.3) \mathrm{m} / \mathrm{s}$ and having a gait speed $<1 \mathrm{~m} / \mathrm{s}$ was prevalent in $39.8 \%$ of the participants. In univariate analyses, the characteristics associated with gait speed were hearing loss, education, hypertension, diabetes, and stroke (Table 1).

Shown in Figure 1, exploratory analyses using a non-parametric smoother demonstrated that both increasing age $(r=-0.20, p<0.001)$ and hearing loss $(r=-0.16, p<.001)$ were relatively weakly associated with gait speed [21]. After adjusting for age, demographic variables (sex, education, race), and cardiovascular risk factors (diabetes, hypertension, smoking, stroke), a $25 \mathrm{~dB}$ increase in hearing loss (analogous to shifting from a normal to a mild hearing loss) was associated with a gait speed difference of $-0.054 \mathrm{~m} / \mathrm{s}(\mathrm{p}<0.01)$. Including individuals with gait speed $<0.6 \mathrm{~m} / \mathrm{s}$ (cutpoint for gait speed as predictor of frailty, functional or cognitive decline) or restricting the analytical cohort to only participants with hearing loss $\leq 40 \mathrm{~dB}$ (thereby excluding those individuals with a moderate to severe hearing loss) did not appreciably affect the findings (Table 2). We also tested whether sex moderated the association of hearing loss with gait speed. In a model adjusted for demographic and cardiovascular risk factors, there was no evidence for an interaction between hearing loss and $\operatorname{sex}(\mathrm{p}=.14)$. An additional sensitivity analysis using gait speed normalized with respect to standing height did not change the results of our findings (data not shown).

To assess the magnitude of the reduction in gait speed associated with hearing loss, we estimated the difference in chronological age that would be equivalent to the association of a $25 \mathrm{~dB}$ increase in hearing thresholds with gait speed. In a fully adjusted model accounting for age, demographic, and cardiovascular risk factors, a one year difference in age was associated with a gait speed difference of $-0.004 \mathrm{~m} / \mathrm{s}(95 \% \mathrm{CI}:-0.007--0.002, \mathrm{p}<0.001)$ while a $25 \mathrm{~dB}$ hearing loss was associated with a gait speed difference of $-0.054 \mathrm{~m} / \mathrm{s}(95 \%$ CI: $-0.090-0.018, \mathrm{p}<0.01)$. Therefore, the difference in age equivalent to the gait speed reduction associated with a $25 \mathrm{~dB}$ shift in hearing loss is approximately 10 years. 
We also investigated the association of hearing aid use with gait speed and found that hearing aid use was not associated with gait speed $(\beta=-0.009 \mathrm{~m} / \mathrm{s}, \mathrm{p}=0.85)$ after adjusting for all covariates (data not shown). However, these results were based on a small number of participants $(n=12)$ who reported hearing aid use and therefore must be interpreted with caution.

We explored the association of hearing loss with gait speed as a dichotomous variable using a gait speed cutoff of $<1 \mathrm{~m} / \mathrm{s}$ or $\geq 1 \mathrm{~m} / \mathrm{s}$ [21]. Results from stepwise logistic models demonstrate that hearing loss was significantly associated with a greater odds of having gait speed $<1 \mathrm{~m} / \mathrm{s}$ in a model adjusted for age, demographic, and cardiovascular risk factors (OR 2.0 [95\% CI: $1.2-3.3$ ] per $25 \mathrm{~dB}$ of hearing loss). These results were not sensitive to including individuals with gait speed $<0.6 \mathrm{~m} / \mathrm{s}$ or restricting the analytical cohort to only those individuals with hearing loss $\leq 40 \mathrm{~dB}$ (Table 3).

\section{Discussion}

In this nationally-representative study of 50-69 year-old adults, greater hearing loss was independently associated with slower observed gait speed. Our results were robust to analyses accounting for multiple confounders and excluding participants with moderate or severe hearing loss. The cross-sectional association of hearing loss with gait speed is clinically significant with a $25 \mathrm{~dB}$ hearing loss (analogous to shifting from a normal to a mild hearing loss) being equivalent to the magnitude of gait speed reduction associated with an age difference of nearly 12 years. A $25 \mathrm{~dB}$ shift in hearing loss was also associated with a 2 -fold increased odds of having a gait speed $<1 \mathrm{~m} / \mathrm{s}$ which is associated with the risk of developing major health-related outcomes including hospitalization and death [22].

Several mechanisms could explain the observed association between hearing loss and gait speed. Pure tone audiometry is considered a measure of the auditory periphery and has been found to be weakly associated with cardiovascular risk factors [23,24]. Therefore, a common pathologic process such as from microvascular disease underlying both hearing loss and slower gait speed is a possibility, but our results were not sensitive to adjustment for factors associated with cardiovascular disease (e.g. diabetes, smoking, hypertension, stroke). There is also the possibility that concomitant pathology of both the cochlear and vestibular sense organs given their shared location within the bony labyrinth of the inner ear [25,26] could explain the association between greater hearing loss and poorer physical mobility and slower gait speed.

Hearing loss could also be associated with gait speed through a mechanistic pathway, possibly mediated by social isolation or cognitive load. Communication impairments caused by hearing loss can lead to progressive social isolation $[9,27]$, and reduced social and physical activities could plausibly mediate the association with slower gait speeds [28]. The effect of hearing loss on cognitive load is suggested by studies demonstrating that under conditions where auditory perception is difficult (i.e. hearing loss), greater cognitive resources are dedicated to auditory perceptual processing at the expense of other cognitive processes such as working memory [6]. Consistent with this hypothesis, hearing loss has been associated with poorer performance on tests of executive function requiring attentional resources $[7,8]$ which are critical for maintaining postural control and balance $[29,30]$ and predict gait speed declines in older adults[3,5].

An interesting finding from this study is that the association of hearing loss with gait speed was present in a cohort of middle-aged to older adults (ages 50-69). Our study did not focus exclusively on older adults because the audiometric testing protocol in NHANES was not performed in individuals $>69$ years during the time period when gait speed was measured. 
This result suggests that the association of hearing loss with gait speed may be present relatively early in mid-adult life and not only in older adults.

In the current study, self-reported hearing aid use among individuals with hearing loss was not associated with gait speed, but there were only a small number $(n=12)$ of participants using hearing aids. The direction of any observed association also could not be established in a cross-sectional study. For example, individuals who are more physically active may also be more likely to obtain hearing aids. Ultimately, investigating causality between hearing rehabilitative treatment and improved physical functioning will require a randomized controlled trial.

A key limitation of this study is the reliance on cross-sectional data rather than on longitudinal trajectories of hearing loss and gait speed over time. Therefore, the findings may be subject to bias by cohort effects or obscured by inter-individual heterogeneity in participant characteristics. However, the restricted age range of the study population (50-69 year-olds) may help limit potential biases introduced by cohort effects. In addition, interindividual heterogeneity in participant characteristics would tend to bias any results toward the null hypothesis whereas our results demonstrated a robust association between hearing loss and gait speed. Residual confounding by other medical factors (e.g. neurological diseases leading to both hearing loss and reduced gait speed) is also possible, but known risk factors for hearing loss and slower gait speed were adjusted for all models.

Gait speed is a robust indicator of health status and a strong predictor of morbidity and mortality in older adults. Therefore, identifying modifiable risk factors for reduced physical vitality and slower gait speed among older adults is a critical public health priority. Further research is needed to investigate the basis of the observed association between hearing loss and slower gait speed and to determine whether these pathways may be amenable to hearing rehabilitative interventions.

\section{Supplementary Material}

Refer to Web version on PubMed Central for supplementary material.

\section{Acknowledgments}

Funding/Support:

This work was supported by the National Institute on Deafness and Other Communication Disorders (1K23DC011279 to F.L.), a Triological Society/American College of Surgeons Clinician Scientist Award (F.L.), the Eleanor Schwartz Charitable Foundation (F.L.), and the intramural research program of the National Institute on Aging (E.S., L.F.).

Role of the Sponsor:

The funding organization had no role in the design and conduct of the study; in the collection, analysis, and interpretation of the data; or in the preparation of the manuscript.

\section{References}

1. Abellan Van Kan G, Rolland Y, Andrieu S, Bauer J, Beauchet O, Bonnefoy M, Cesari M, Donini LM, Gillette-Guyonnet S, Inzitari M. Gait speed at usual pace as a predictor of adverse outcomes in community-dwelling older people an International Academy on Nutrition and Aging (IANA) Task Force. The Journal of Nutrition, Health and Aging. 2009; 13:881-889.

2. Studenski S, Perera S, Patel K, Rosano C, Faulkner K, Inzitari M, Brach J, Chandler J, Cawthon P, Connor EB. Gait speed and survival in older adults. JAMA: the journal of the American Medical Association. 2011; 305:50-58. [PubMed: 21205966] 
3. Atkinson HH, Rosano C, Simonsick EM, Williamson JD, Davis C, Ambrosius WT, Rapp SR, Cesari M, Newman AB, Harris TB. Cognitive function, gait speed decline, and comorbidities: the health, aging and body composition study. The Journals of Gerontology Series A: Biological Sciences and Medical Sciences. 2007; 62:844-850.

4. Ijmker T, Lamoth CJC. Gait and cognition: The relationship between gait stability and variability with executive function in persons with and without dementia. Gait \& Posture. 2012; 35:126-130. [PubMed: 21964053]

5. Watson NL, Rosano C, Boudreau RM, Simonsick EM, Ferrucci L, Sutton-Tyrrell K, Hardy SE, Atkinson HH, Yaffe K, Satterfield S. Executive function, memory, and gait speed decline in wellfunctioning older adults. The Journals of Gerontology Series A: Biological Sciences and Medical Sciences. 2010; 65:1093-1100.

6. Tun PA, McCoy S, Wingfield A. Aging, hearing acuity, and the attentional costs of effortful listening. Psychology and Aging. 2009; 24:761-766. [PubMed: 19739934]

7. Lin FR. Hearing Loss and Cognition Among Older Adults in the United States. The Journals of Gerontology Series A: Biological Sciences and Medical Sciences. 2011; 66:1131-1136.

8. Lin FR, Ferrucci L, Metter EJ, An Y, Zonderman AB, Resnick SM. Hearing loss and cognition in the Baltimore longitudinal study of aging. Neuropsychology. 2011; 25:763-770. [PubMed: 21728425]

9. Strawbridge WJ, Wallhagen MI, Shema SJ, Kaplan GA. Negative consequences of hearing impairment in old age. The Gerontologist. 2000; 40:320-326. [PubMed: 10853526]

10. Lin FR, Niparko JK, Ferrucci L. Hearing Loss Prevalence in the United States. Arch Intern Med. 2011; 171:1851-1852. [PubMed: 22083573]

11. Chien W, Lin FR. Prevalence of Hearing Aid Use Among Older Adults in the United States. Arch Intern Med. 2012; 172:292. [PubMed: 22332170]

12. Centers for Disease Control and Prevention (CDC). National Center for Health Statistics (NCHS). National Health and Nutrition Examination Survey. Hyattsville, MD: U.S. Department of Health and Human Services, Centers for Disease Control and Prevention; 2010. http://www.cdc.gov/nchs/ nhanes.htm/.

13. Centers for Disease Control. [Accessed 9/12/2011] Audiometry/Tympanometry Procedures Manual. http://www.cdc.gov/nchs/data/nhanes/au.pdf.

14. World Health Organization Prevention of Blindness and Deafness (PBD) Program. Prevention of Deafness and Hearing Impaired Grades of Hearing Impairment. http://www.who.int/pbd/deafness/ hearing_impairment_grades/en/index.html.

15. Clark JG. Uses and abuses of hearing loss classification. ASHA. 1981; 23:493-500. [PubMed: 7052898]

16. Centers for Disease Control and Prevention (CDC). [Accessed 9/22/2011] NHANES Muscular Strength Examination: Component Description. http://www.cdc.gov/nchs/nhanes/ nhanes1999-2000/MSX.htm.

17. NHANES Analytic and Reporting Guidelines. www.cdc.gov/nchs/data/nhanes/nhanes_03_04/ nhanes_analytic_guidelines_dec_2005.pdf.

18. Fried LP, Tangen CM, Walston J, Newman AB, Hirsch C, Gottdiener J, Seeman T, Tracy R, Kop WJ, Burke G. Frailty in older adults. The Journals of Gerontology Series A: Biological Sciences and Medical Sciences. 2001; 56:M146-M157.

19. Hof AL. Scaling gait data to body size. Gait \& Posture. 1996; 4:222-223.

20. Stansfield BW, Hillman SJ, Hazlewood ME, Robb JE. Regression analysis of gait parameters with speed in normal children walking at self-selected speeds. Gait \& posture. 2006; 23:288-294. [PubMed: 15978813]

21. Taylor T. Interpretation of the correlation coefficient: a basic review. JDMS. 1990; 1:35-39.

22. Cesari M, Kritchevsky SB, Penninx BWHJ, Nicklas BJ, Simonsick EM, Newman AB, Tylavsky FA, Brach JS, Satterfield S, Bauer DC. Prognostic Value of Usual Gait Speed in Well-Functioning Older People--Results from the Health, Aging and Body Composition Study. Journal of the American Geriatrics Society. 2005; 53:1675-1680. [PubMed: 16181165] 
23. Cruickshanks KJ, Klein R, Klein BEK, Wiley TL, Nondahl DM, Tweed TS. Cigarette smoking and hearing loss. JAMA: the journal of the American Medical Association. 1998; 279:1715-1719. [PubMed: 9624024]

24. Dalton DS, Cruickshanks KJ, Klein R, Klein BE, Wiley TL. Association of NIDDM and hearing loss. Diabetes Care. 1998; 21:1540-1544. [PubMed: 9727906]

25. Agrawal Y, Carey JP, Della Santina CC, Schubert MC, Minor LB. Disorders of balance and vestibular function in US adults: data from the National Health and Nutrition Examination Survey, 2001-2004. Archives of Int Med. 2009; 169:938.

26. Baloh RW, Enrietto J, Jacobson KM, Lin A. Age-Related Changes in Vestibular Function. Annals of the New York Academy of Sciences. 2001; 942:210-219. [PubMed: 11710463]

27. Nachtegaal J, Smit JH, Smits CAS, Bezemer PD, van Beek JHM, Festen JM, Kramer SE. The association between hearing status and psychosocial health before the age of 70 years: results from an internet-based national survey on hearing. Ear and Hearing. 2009; 30:302-312. [PubMed: 19322094]

28. Kuo HK, Leveille SG, Yen CJ, Chai HM, Chang CH, Yeh YC, Yu YH, Bean JF. Exploring how peak leg power and usual gait speed are linked to late-life disability: data from the National Health and Nutrition Examination Survey (NHANES), 1999-2002. American Journal of Physical Medicine \& Rehabilitation/Association of Academic Physiatrists. 2006; 85:650-658. [PubMed: 16865019]

29. Shumway-Cook A, Woollacott M. Attentional demands and postural control: the effect of sensory context. Journals of Gerontology-Biological Sciences and Medical Sciences. 2000; 55:M10-M16.

30. Woollacott M, Shumway-Cook A. Attention and the control of posture and gait: a review of an emerging area of research. Gait \& posture. 2002; 16:1-14. [PubMed: 12127181] 


\section{Highlights}

In a study of 1,180 adults ages 50-69 years, greater hearing loss was independently associated with slower gait speed.

The mechanistic basis of this association is unknown

Studies investigating whether hearing rehabilitative interventions could affect gait and physical functioning are needed. 

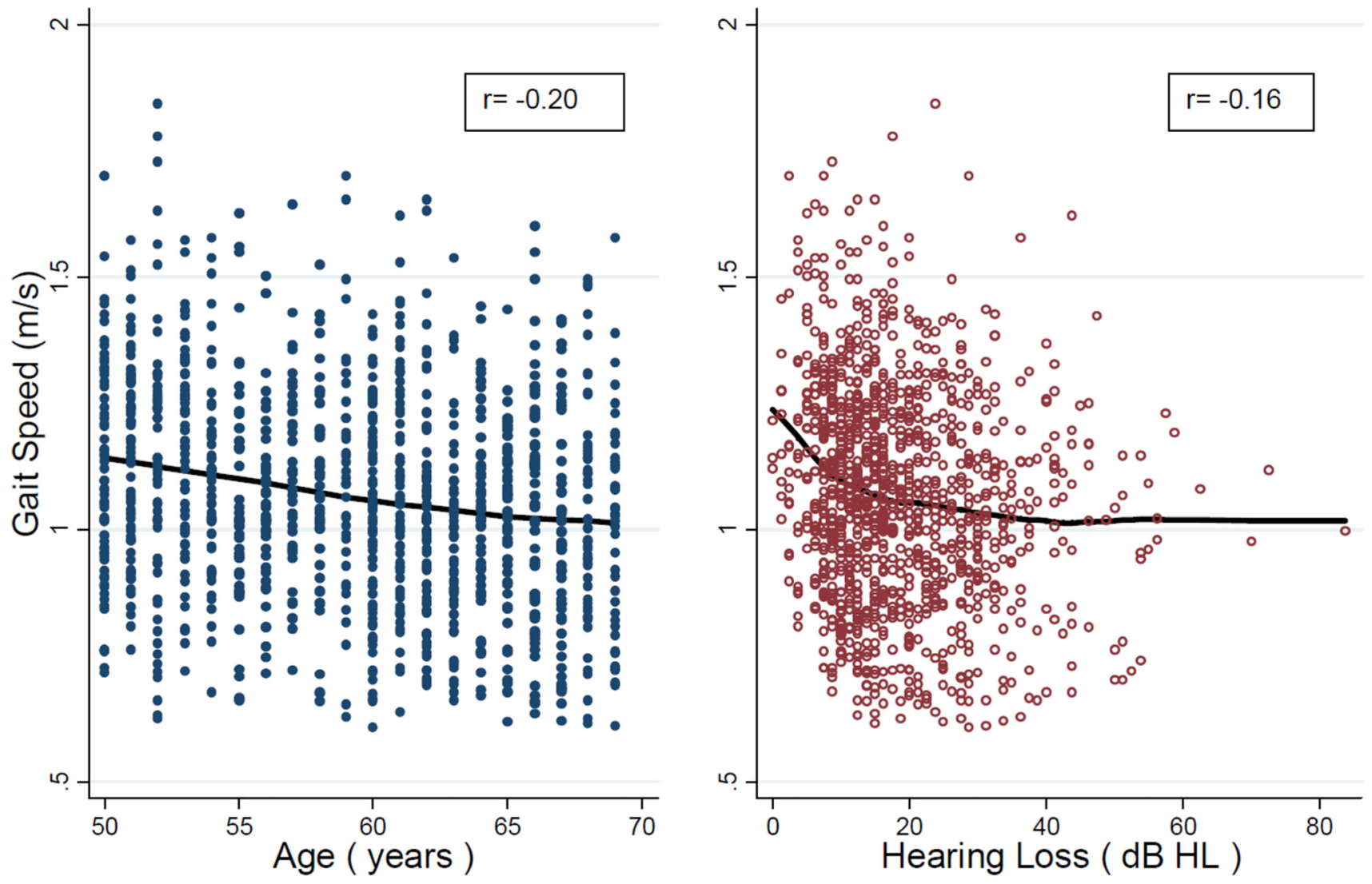

Figure 1.

Cross-sectional association of age and hearing loss with gait speed in participants aged 5069 years, NHANES 1999-2002. 
Table 1

Demographic characteristics of participants aged 50-69 years with assessment of audiometric hearing and gait speed, National Health and Nutritional Examination Surveys (NHANES), 1999-2002

\begin{tabular}{|c|c|c|c|}
\hline Characteristics & $\begin{array}{c}\text { Cohort } \\
(N=1180)\end{array}$ & $\begin{array}{c}\text { Univariate } \\
\text { mean gait speed } \\
(\mathbf{m} / \mathbf{s}) \text {, S.D. }\end{array}$ & \\
\hline Age, mean years (S.D.) & $59.4(5.8)$ & --- & \\
\hline Hearing loss, mean dB HL (S.D) & $18.6(11.1)$ & --- & \\
\hline \multicolumn{4}{|l|}{ Hearing loss category, n (\%) } \\
\hline Normal ( $\leftrightharpoons 25 \mathrm{~dB}$ ) & $909(77.0)$ & $1.06,0.26$ & $<0.001$ \\
\hline Mild (26-40 dB) & $210(17.8)$ & $1.00,0.23$ & \\
\hline Moderate (41-70dB) & $59(5.0)$ & $0.98,0.25$ & \\
\hline Severe $(\geq 71 \mathrm{~dB})$ & $2(0.2)$ & $1.06,0.08$ & \\
\hline Hearing aid use, $\mathrm{n}(\%)^{a}$ & $12(4.4)$ & --- & \\
\hline Gait speed, mean m/sec (S.D.) & $1.05(0.3)$ & --- & \\
\hline$<1 \mathrm{~m} / \mathrm{s}, \mathrm{n}(\%)$ & $470(39.8)$ & --- & \\
\hline$\geq 1 \mathrm{~m} / \mathrm{s}, \mathrm{n}(\%)$ & $710(60.2)$ & --- & \\
\hline \multicolumn{4}{|l|}{ Race/ethnicity, n (\%) } \\
\hline White & $599(50.8)$ & $1.12,0.25$ & 0.57 \\
\hline Black & $233(19.7)$ & $0.96,0.22$ & \\
\hline Hispanic & $311(26.4)$ & $0.98,0.24$ & \\
\hline Other & $37(3.1)$ & $1.08,0.23$ & \\
\hline \multicolumn{4}{|l|}{ Education, n (\%) } \\
\hline$<12^{\text {th }}$ grade & $420(35.6)$ & $0.94,0.24$ & $<0.001$ \\
\hline High school graduate & 255 (21.6) & $1.06,0.27$ & \\
\hline Some college & $258(21.9)$ & $1.09,0.22$ & \\
\hline College graduate & $246(20.9)$ & $1.19,0.22$ & \\
\hline \multicolumn{4}{|l|}{ Smoking, n (\%) } \\
\hline Never & $505(42.9)$ & $1.05,0.25$ & 0.40 \\
\hline Former & $434(36.8)$ & $1.08,0.28$ & \\
\hline Current & $239(20.3)$ & $1.02,0.22$ & \\
\hline \multicolumn{4}{|l|}{ Hypertension } \\
\hline Yes & $426(36.3)$ & $1.01,0.28$ & $<0.001$ \\
\hline No & 747 (63.7) & $1.07,0.24$ & \\
\hline \multicolumn{4}{|l|}{ Diabetes, n (\%) } \\
\hline Yes & $181(15.3)$ & $0.94,0.24$ & $<0.001$ \\
\hline No & 999 (84.7) & $1.07,0.25$ & \\
\hline \multicolumn{4}{|l|}{ Stroke, n (\%) } \\
\hline Yes & $39(3.3)$ & $0.94,0.23$ & $<0.01$ \\
\hline No & $1140(96.7)$ & $1.05,0.26$ & \\
\hline
\end{tabular}




\section{Table 2}

Stepwise regression models of gait speed per $25 \mathrm{~dB}$ of hearing loss in participants aged 50-69 years, NHANES 1999-2002

\begin{tabular}{|c|c|c|}
\hline & $\mathbf{N}$ & $\begin{array}{c}\text { Hearing loss } \\
\beta^{a} \text { per } 25 \mathrm{~dB} \\
(95 \% \mathrm{CI})\end{array}$ \\
\hline Base model (hearing loss + age only) & 1138 & $-0.054^{* *}(-0.090$ to -0.019$)$ \\
\hline Base + demographic factors $b$ & 1137 & $-0.059^{* *}(-0.096$ to -0.021$)$ \\
\hline Base + demographic factors + cardiovascular risk factors $c$ & 1127 & $-0.054^{* *}(-0.090$ to -0.018$)$ \\
\hline $\begin{array}{l}\text { Base + demographic factors + cardiovascular risk factors + restricted to participants with hearing loss } \leq \\
40 \mathrm{~dB}\end{array}$ & 1072 & $-0.058 *(-0.109$ to -0.006$)$ \\
\hline $\begin{array}{l}\text { Base }+ \text { demographic factors }+ \text { cardiovascular risk factors }+ \text { including participants with gait speed }< \\
0.06 \mathrm{~m} / \mathrm{s}\end{array}$ & 1167 & $-0.056^{* *}(-0.092$ to -0.021$)$ \\
\hline
\end{tabular}

$a_{\beta \text {-coefficient represents the expected difference in gait speed associated with a } 25 \mathrm{~dB} \text { increase in hearing loss (analogous to shifting from normal }}$ hearing to mild hearing loss). Negative $\beta$ 's indicate slower gait speed with increasing hearing loss.

${ }^{b}$ Demographic factors include sex, race/ethnicity, and education.

${ }^{c}$ Cardiovascular risk factors include smoking status, hypertension, diabetes mellitus, and stroke.

$*$

p $<0.05$.

**

$\mathrm{p}<0.01$. 


\section{Table 3}

Stepwise logistic regression models of gait speed as a dichotomous variable $(<1 \mathrm{~m} / \mathrm{s}$ vs $\geq 1 \mathrm{~m} / \mathrm{s})$ per $25 \mathrm{~dB}$ of hearing loss, NHANES 1999-2002.

\begin{tabular}{|c|c|c|}
\hline & $\mathbf{N}$ & $\begin{array}{c}\text { Odds of Gait Speed }<1 \mathrm{~m} / \mathrm{s} \\
\text { per } 25 \mathrm{~dB} \text { of Hearing Loss } \\
(95 \% \mathrm{CI})\end{array}$ \\
\hline Base model (hearing loss + age only) & 1138 & $1.7^{*}(1.1-2.5)$ \\
\hline Base + demographic factors $a$ & 1137 & $2.0^{*}(1.2-3.3)$ \\
\hline Base + demographic factors + cardiovascular risk factors $b$ & 1127 & $2.0^{* *}(1.2-3.3)$ \\
\hline $\begin{array}{l}\text { Base }+ \text { demographic factors }+ \text { cardiovascular risk factors }+ \text { restricted to participants with hearing loss } \leq \\
40 \mathrm{~dB}\end{array}$ & 1072 & $2.3^{*}(1.2-4.6)$ \\
\hline $\begin{array}{l}\text { Base + demographic factors }+ \text { cardiovascular risk factors + Including participants with gait speed }<0.6 \\
\mathrm{~m} / \mathrm{s}\end{array}$ & 1167 & $2.0^{* *}(1.2-3.2)$ \\
\hline
\end{tabular}

${ }^{a}$ Demographic factors include sex, race/ethnicity, and education.

${ }^{b}$ Cardiovascular risk factors include smoking status, hypertension, diabetes mellitus, and stroke.

$*$

p $<0.05$.

$* *$

$\mathrm{p}<0.01$. 